A N N A LES

UNIVERSITATIS M ARIAE CURIE-SKŁODOWSKA

LUBLIN - POLONIA

VOL. LXVI, 1, 2

SECTIO AA

2011

\title{
Solute permeation through lipid membrane: Statistical Rate Theory approach
}

\author{
W. Piasecki ${ }^{1, *}$ and R. Charmas ${ }^{2}$ \\ ${ }^{1}$ Department of Biochemistry, Faculty of Physical Education \\ and Sport in Biala Podlaska \\ The Josef Pilsudski University of Physical Education in Warsaw, Poland \\ ${ }^{2}$ The State College of Computer Science \\ and Business Administration in Łomża, Poland
}

\begin{abstract}
A new model describing transport across lipid membrane was developed based on the Statistical Rate Theory (SRT) of interfacial transfer. In our calculations we replaced lipid membrane with well-defined octanol membrane. According to SRT the rate of solute transfer across water/octanol interface depends on the ratio of solute concentration in the both phases and the water/octanol partition coefficient. In the case of thick membranes and thick diffuse layers our model predicts almost linear dependence of flux on concentration gradient. However, for thin membranes and thin diffuse layers flux becomes nonlinear function of concentration gradient, especially for more polar solutes and higher gradients.
\end{abstract}

\section{INTRODUCTION}

It has been known from over 100 years that the lipophilicity of compound is a major factor governing its transfer across lipid membrane [1, 2]. Because biological membranes are not convenient to physicochemical studies, substitute substances which properties resemble lipid membrane were frequently used in

\footnotetext{
${ }^{*}$ Corresponding author: Wojciech Piasecki, Department of Biochemistry, Faculty of Physical Education and Sport in Biala Podlaska, The Josef Pilsudski University of Physical Education in Warsaw, ul. Akademicka 2, 21-500 Biala Podlaska, Poland, Tel. +48 83 3428729, Fax. +48 83 3428800, e-mail: wojciech.piasecki@ awf-bp.edu.pl
} 
many experiments. The most popular substance of this type is octanol. The common measure of compound lipophilicity is octanol/water partition coefficient $P_{o w}$. Its values were determined for thousands of compounds used in pharmacological, toxicological and cosmetic research.

The common practice in these studies was the assumption that the transport of solutes through lipid membrane was governed by passive diffusion [3]. However there are evidences that interfacial transfer can be rate-limiting step during membrane permeation $[4,5]$. There are several experimental studies dealing with the kinetics of the partitioning between water and octanol. Their critical discussion can be found in the review written by Fisk et al. [6].

The rate constants of interfacial transport can be determined by using different experimental techniques such as falling drops, horizontal side-by-side diffusion cells, or rotating diffusion cell method [7-9]. The interpretation of the results of those experiments was based on the linear nonequlibrium thermodynamics of membrane transport [10]. In this approach one assumes that solute flux through membrane $\left[\mathrm{mol} \mathrm{m} \mathrm{m}^{-2} \mathrm{~s}^{-1}\right]$ linearly depends on the concentration difference on the both sides of membrane. In this paper we would like to apply the Statistical Rate Theory of interfacial transport (SRT) to describe the kinetics of the partitioning of solute between octanol and water.

The SRT approach was developed by Ward and it is based on the local thermodynamic equilibrium assumption and relates the transfer kinetics with the quantum mechanical probability of crossing the interface by a single particle $[11,12]$. The driving force for molecule transfer in the SRT is the change in the microscopic states distributions, which is associated with the transfer entropy (or equivalently with the gradient of chemical potential in both phases).

The SRT was successfully applied to describe the transport rates of various interfacial processes like liquid evaporation [13], gas adsorption on solids [14], the permeation of ionic channels in biological membranes [15], the surfactant adsorption at liquid interfaces [16], metal ion adsorption on biosorbents [17], and the relaxation time of proton adsorption from solution on oxides [18].

In the next section we will develop the equations based on the SRT approach for the rate of solute transport across octanol membrane. Next, we will compare the SRT predictions with the results given by the classical linear model for two compounds soluble in water but having different polarity, caffeine and butanol.

\section{THEORY}

According to the Statistical Rate Theory (SRT) the rate of interfacial transport depends on the difference of solute chemical potential in two phases (e.g. octanol and water phase) as follows, 


$$
J_{o w}=R_{e x}\left[\exp \left(\frac{\mu_{o}-\mu_{w}}{R T}\right)-\exp \left(\frac{\mu_{w}-\mu_{o}}{R T}\right)\right]
$$

where $J_{o w}$ is the net flux through interface, and $\mu_{o}$ and $\mu_{w}$ are the chemical potentials of the solute in octanol and water phase, respectively. $R_{e x}$ is the exchange rate between two phases at equilibrium.

The SRT is based on the assumption of local thermodynamic equilibrium, so we can use chemical potentials in eq (1), however in the SRT we do not assume that investigated system is near equilibrium. Equation (1) describes two transport processes: flux from octanol to water and from water to octanol. In the equilibrium total flux is equal to zero.

Starting from the following expressions for solute chemical potential in both phases,

$$
\begin{aligned}
& \mu_{o}=\mu_{o}^{0}+R T \ln a_{o} \\
& \mu_{w}=\mu_{w}^{0}+R T \ln a_{w}
\end{aligned}
$$

where $\mu_{o}^{0}$ and $a_{o}$ denote standard chemical potential and the activity of the solute in octanol phase, and $\mu_{w}^{0}$ and $a_{w}$ denote the same quantities in water phase, we can transform the equation (1) into,

$$
J_{o w}=R_{e x}\left[\exp \left(\frac{\mu_{o}^{0}-\mu_{w}^{0}}{R T}\right) \frac{a_{o}}{a_{w}}-\exp \left(\frac{\mu_{w}^{0}-\mu_{o}^{0}}{R T}\right) \frac{a_{w}}{a_{o}}\right]
$$

Taking into account the thermodynamic definition of the partition coefficient $P_{o w}$

$$
P_{o w}=\exp \left(\frac{\mu_{w}^{0}-\mu_{o}^{0}}{R T}\right)
$$

and replacing activities by concentrations we finally obtain,

$$
J_{o w}=R_{e x}\left[\frac{c_{o}}{P_{o w} c_{w}}-\frac{P_{o w} c_{w}}{c_{o}}\right]
$$


The equation (5) describes transfer kinetics through octanol/water interface according to the SRT. This equation predicts that flux through interface depends on solute concentration ratio in both phases, partition coefficient $P_{o w}$ and the exchange rate at equilibrium between organic and aqueous phase $R_{e x}$. The Statistical Rate Theory predicts nonlinear dependence of flux on the concentrations in both phases.

According to the classical description of solute transport kinetics through liquid interface the flux depends linearly on the concentrations in the both phases $[6,7]$.

$$
J_{o w}=k_{o w} c_{o}-k_{w o} c_{w}
$$

where $k_{o w}$ is the rate constant for crossing the interface from octanol to water and $k_{w o}$ is the reverse. The rate constants $k_{o w}$ and $k_{w o}$ are related with the partition coefficient by the relation $P_{o w}=k_{w o} / k_{o w}$.

The analysis presented above is the starting point for kinetic description of the system depicted in Figure 1. There are two compartments containing aqueous solutions separated by membrane which pores are completely filled with octanol. Such system is often used to investigate skin permeability (where a solute is dissolved in aqueous vehicle applied on the skin and penetrate through stratum corneum to viable epidermis) [19,20].

A solute which cross the membrane has to overcome three diffusion barriers (two in aqueous phase and one in octanol) and two interfaces: water-octanol and octanol-water. When bulk phases are well stirred the drop of concentration profile can only be observed in thin layers adjacent to water/octanol boundary. The solute transport in this layers is due to passive diffusion. In steady-state condition concentration gradients in diffuse layers are linear and the following equations based on the Fick's first law of diffusion can be written:

$$
\begin{aligned}
& J_{w 1}=\frac{D_{w}}{h_{w}}\left(c_{w 1 b}-c_{w 1}\right) \\
& J_{o}=\alpha \frac{D_{0}}{h_{o}}\left(c_{o 1}-c_{o 2}\right) \\
& J_{w 2}=\frac{D_{w}}{h_{w}}\left(c_{w 2}-c_{w 2 b}\right)
\end{aligned}
$$


where $\alpha$ denotes porosity of membrane (other symbols are defined in the description of Figure 1).

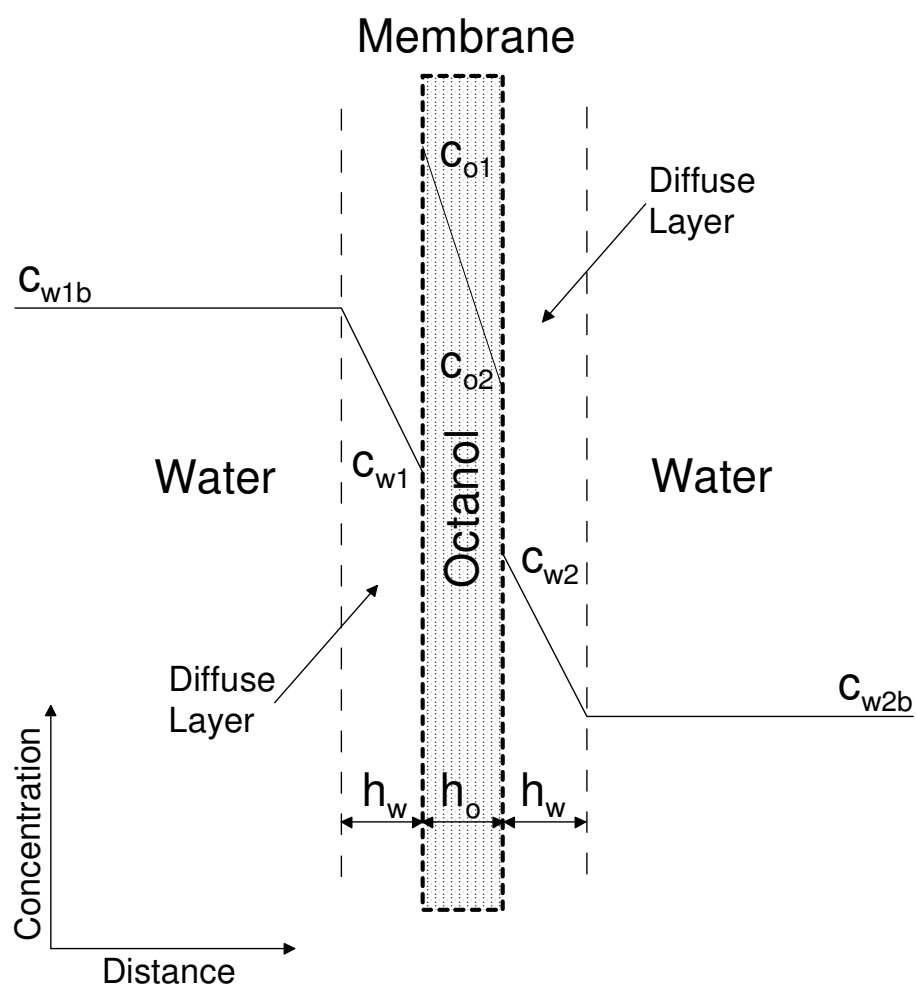

Fig. 1. Two aqueous solutions separated by octanol membrane. Bulk concentrations of substance in two water compartments are denoted by $c_{w 1 b}$ and $c_{w 2 b}$, the concentrations at outer and inner membrane interfaces are denoted by $c_{w 1}$ and $c_{w 2}$, and $c_{o 1}$ and $c_{o 2}$. $h_{w}$ is the thickness of diffuse layer in water and $h_{o}$ the thickness of octanol membrane, which porosity (i.e. fraction of free volume in membrane) is $\alpha$.

According to the Statistical Rate Theory fluxes through water-octanol and octanol-water interface can be expressed as,

$$
J_{w o}=\alpha R_{e x}\left[\frac{P_{o w} c_{w 1}}{c_{o 1}}-\frac{c_{o 1}}{P_{o w} c_{w 1}}\right]
$$




$$
J_{o w}=\alpha R_{e x}\left[\frac{c_{o 2}}{P_{o w} c_{w 2}}-\frac{P_{o w} c_{w 2}}{c_{o 2}}\right]
$$

Applying the classical approach we can write [6],

$$
\begin{aligned}
& J_{w o}=\alpha\left(k_{w o} c_{w 1}-k_{o w} c_{o 1}\right) \\
& J_{o w}=\alpha\left(k_{o w} c_{o 2}-k_{w o} c_{w 2}\right)
\end{aligned}
$$

In steady-state all fluxes defined above are equal to $J_{s t}$. The equations (7abc) and (8ab) can be combined in one expression,

$$
J_{s t}=\frac{\alpha D_{o} P_{o w}}{2 h_{o}}\left[\sqrt{\left(\left(\frac{J_{s t}}{\alpha R_{e x}}\right)^{2}+4\right)} \times\left(\left(c_{w 1 b}-c_{w 2 b}\right)-\frac{2 J_{s t} h_{w}}{D_{w}}\right)-\frac{J_{s t}}{\alpha R_{e x}}\left(c_{w 1 b}+c_{w 2 b}\right)\right]
$$

The equation (10) which is based on the Statistical Rate Theory of interfacial transport is nonlinear equation with reference to the flux and concentrations and can be solved numerically.

The equations (7abc) and (9ab) can also be joined in one expression, which is the standard formula used to calculate flux across lipid membrane,

$$
J_{s t}=\frac{P_{o w} \cdot\left(c_{w 1 b}-c_{w 2 b}\right)}{\frac{2 P_{o w} \cdot h_{w}}{D_{w}}+\frac{h_{o}}{\alpha D_{o}}+\frac{2}{\alpha k_{o w}}}
$$

In equation (11) driving force for solute transport is the concentration difference in the numerator of eq (11). The flux resistance expressed by the denominator of eq (11) is divided into individual transport resistances due to the diffusion through unstirred diffuse layers in aqueous and organic phases and the interfacial transfer.

\section{RESULTS AND DISCUSSION}

So far the interfacial transfer constants have been determined by using the standard interfacial transport model assuming linear dependence between solute concentration and its flux across membrane and applying the transport resistance summation approach $[4,5,7]$. Because in literature we could only find kinetic parameters determined by using the above approach (without publication of raw 
experimental data), we have decided to check the Statistical Rate Theory predictions in the following way.

We decided to compare fluxes through octanol membrane (depicted in Figure 1) predicted by two approaches presented in the Theory section. We used the equations (10) and (11) to calculate the flux as a function of solute concentration in one of aqueous phases. It was assumed that the volumes of two aqueous phase are the same and equal to $100 \mathrm{~cm}^{3}$. The total amount of substance dissolved in the system was equal to $10^{-2}$ mole. Additionally, it was assumed that in the starting point of our calculations $90 \%$ of solute was presented in one compartment and the rest (10\%) was dissolved in the second. This initial separation of solute between two aqueous compartments was necessary because the our derivation of transport equations was done assuming steady-state condition.

Calculations were performed for two compounds with different lipophilicity: butanol and caffeine with partition coefficients $P_{o w}=7.83$ and $P_{o w}=0.68$, respectively. These compounds were chosen because according to Fisk et al. for substances with $\log P_{o w}$ below 1.2 the kinetics of interfacial transfer should control the overall rate of transport [6]. The other parameters used in calculations were taken from Mosher's paper [7] and collected in Table 1. We chose Mosher's data because they were obtained using side-by-side diffusion cells setup, in which one can control interfacial area and hydrodynamics of the system. Additionally, similar setups are commercially available and used to determine the permeability of drugs and cosmetic ingredients across the skin.

Figure 2 depicts butanol and caffeine fluxes through octanol membrane with thickness $h_{o}=100 \mu \mathrm{m}$ and porosity $\alpha=0.75$ as a function of bulk concentration of solute in one of aqueous phases. The volume of octanol in membrane is small enough, so it can be neglected in the mass balance of solute (for solutes with $\log P_{o w}<1.2$, which are considered in this paper).

As it follows from Figure 2 the both models predict identical butanol flux in the whole concentration range. For caffeine, which is more polar compound than butanol, we observe deviation of SRT flux from linearity for higher concentration gradient. We assumed that the thickness of unstirred diffusion layer in aqueous solution was equal to $h_{w}=200 \mu \mathrm{m}$.

This value is consistent with Mosher's estimations. He found that unstirred diffusion layer in aqueous solution had thickness $h_{w}=176 \pm 56 \mu \mathrm{m}$, and the thickness of stagnant diffusion layer in octanol was equal to $h_{o}=97.6 \pm 9.6 \mu \mathrm{m}$ [7]. On the other hand Miller, using falling drops technique, estimated that $h_{w}=9.1 \mu \mathrm{m}$ and $h_{o}=1.2 \mu \mathrm{m}$ [21]. 
Tab. 1. The parameters values used in the calculations. The data were taken from Mosher's paper [7] or assumed by us. In calculations it was assumed that two aqueous compartments had the same volumes $\left(100 \mathrm{~cm}^{3}\right)$ and total amount of butanol or caffeine in the system was equal to $10^{-2}$ moles.

\begin{tabular}{|c|c|c|}
\hline Parameter & Butanol & Caffeine \\
\hline Octanol-water partition coefficient $P_{o w}$ & 7.83 & 0.68 \\
\hline Diffusion constant in water $D_{w}\left[\times 10^{-6} \mathrm{~cm}^{2} \mathrm{~s}^{-1}\right]$ & 10.4 & 7.21 \\
\hline Diffusion constant in octanol $D_{o}\left[\times 10^{-6} \mathrm{~cm}^{2} \mathrm{~s}^{-1}\right]$ & 2.04 & 1.53 \\
\hline $\begin{array}{c}\text { Thickness of unstirred diffusion layer in water } h_{w}[\mu \mathrm{m}] \\
\text { (assumed) }\end{array}$ & 200 or 20 & 200 or 20 \\
\hline Membrane thickness $h_{o}[\mu \mathrm{m}]($ assumed) & 100 or 10 & 100 or 10 \\
\hline Membrane porosity (assumed) & 0.75 & 0.75 \\
\hline Water-to-octanol rate constant $k_{w o}\left[\mu \mathrm{m} \cdot \mathrm{s}^{-1}\right]$ & 16.6 & 0.834 \\
\hline Octanol-to-water rate constant $k_{o w}\left[\mu \mathrm{m} \cdot \mathrm{s}^{-1}\right]$ & 2.12 & 1.23 \\
\hline $\begin{array}{c}\text { The exchange rates in equilibrium } R_{e x}\left[\mathrm{~mol} \cdot \mathrm{m}^{-2} \cdot \mathrm{s}^{-1}\right] \\
\text { determined in this paper by using the SRT for octanol } \\
\text { membrane separating two aqueous solutions }\end{array}$ & $4.0 \times 10^{-4}$ & $2.0 \times 10^{-5}$ \\
\hline
\end{tabular}

Review of the literature reveals that the thickness of stagnant diffusion layers constitutes significant uncertainty in the determination of kinetic parameters for octanol/water interface.

For a long time it had been believed that the solute transfer rate across lipid membrane is determined by the diffusion near the interface and within the membrane, however the cases in which the interfacial transfer kinetics determined the overall passage rate were reported. For example, Guy and Honda found significant energy barrier for the interfacial transfer of methyl nicotinate across octanol membrane [5]. Next, Miller showed the experimental evidences that the interfacial transfer is a rate-limiting step during passive membrane permeation [8], however his interpretation was immediately criticized by Hladky [22]. After a few years, Miller responded in a more detailed experimental study, which confirmed his initial suggestions, that the interfacial transfer can be the bottle-neck for solute flux across membrane [4]. 

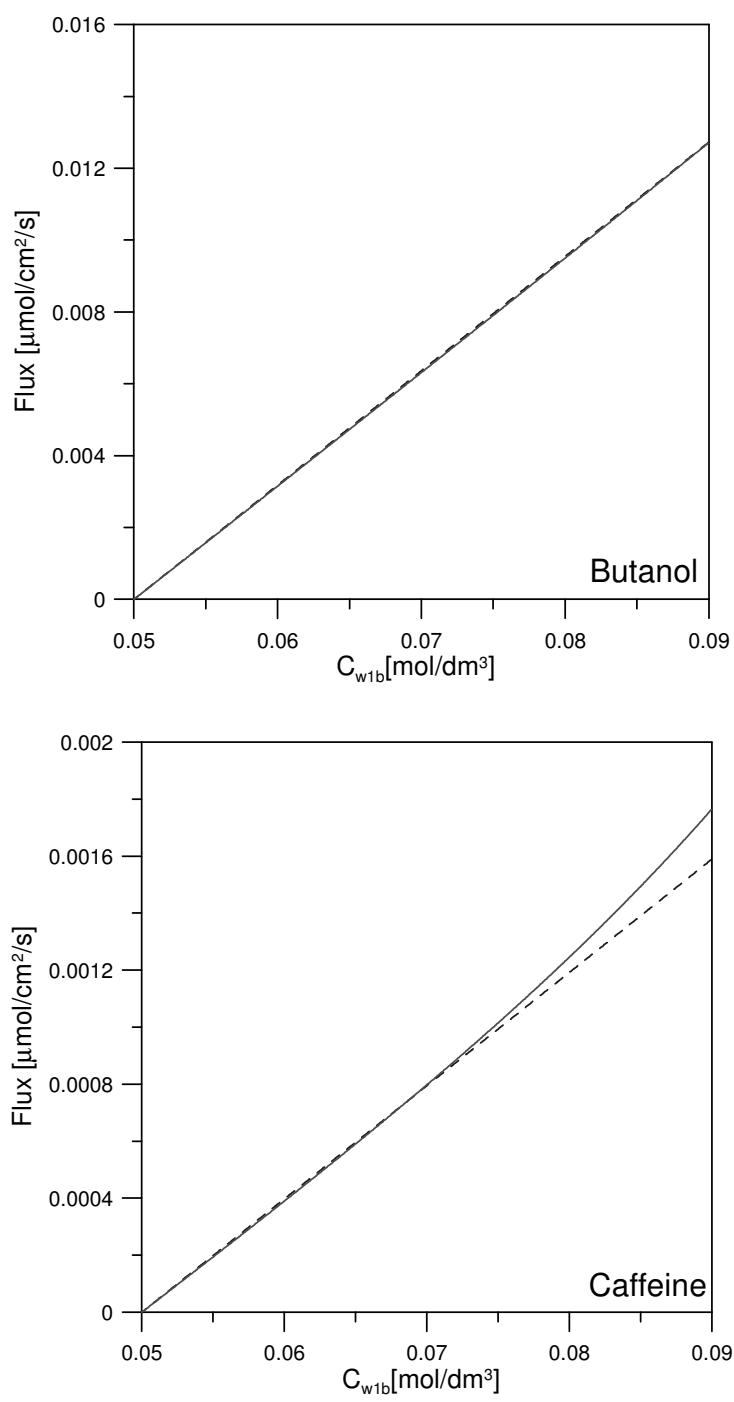

Fig. 2. The fluxes predicted by the SRT using eq (10) (solid red line) and the classical approach using eq (11) (dashed blue line) for butanol and caffeine transport across octanol membrane as a function of bulk concentration of solute in one of aqueous phases. The parameters used in the calculations were collected in Table 1. It was assumed that the thickness of unstirred diffusion layer in aqueous solution is equal to $h_{W}=200 \mu \mathrm{m}$, and membrane thickness is equal to $h_{o}=100 \mu \mathrm{m}$.

The determined thickness of unstirred diffusion layer can change depending on applied experimental technique. In rotating diffusion cell experiments the 
thickness of the diffusion layer depends on the square root of the rotation speed and can be calculated using Levich equation [6].

Recently the application of scanning ion-sensitive microelectrodes has enabled the determination of concentration profiles of permeating ion species [23]. For example for sodium and calcium ions unstirred layer thickness was found to be higher than $100 \mu \mathrm{m}$.

These discrepancies in the estimations of unstirred diffusion layer thickness (about one order of magnitude) may be of great importance for solute flux across interface.

When we assumed that octanol membrane was ten times thinner than before and diffuse layer thickness in water was equal to $h_{w}=20 \mu \mathrm{m}$, we obtained the fluxes depicted in Figure 3. Both for caffeine and butanol we observe that SRT flux becomes nonlinear and greater than the flux predicted by eq (11) for solute concentrations above $0.07 \mathrm{M}$.

The analysis of all results for caffeine and butanol suggests that interfacial transfer for more polar compounds (like caffeine) should exhibits more nonlinear behaviour in comparison with more lipophilic compounds (like butanol). This is probably the result of higher energetic barrier for the transfer of polar compounds across water/octanol interface.

\section{CONCLUSIONS}

The Statistical Rate Theory predicts that the solute flux across octanol membrane is nonlinear function of concentration gradient. The calculated rates of permeation for caffeine and butanol suggest that nonlinear behaviour should be more visible for more polar compounds. Thick unstirred diffuse layers adjacent to the interface smooth out nonlinear dependence of solute flux predicted by SRT and therefore the results obtained are similar to those given by the linear model of membrane permeation.

The SRT formalism, which was developed here, is quite general and can be used to interpret solute flux across any lipid membrane (octanol, silicon, isopropyl myristate etc.). 

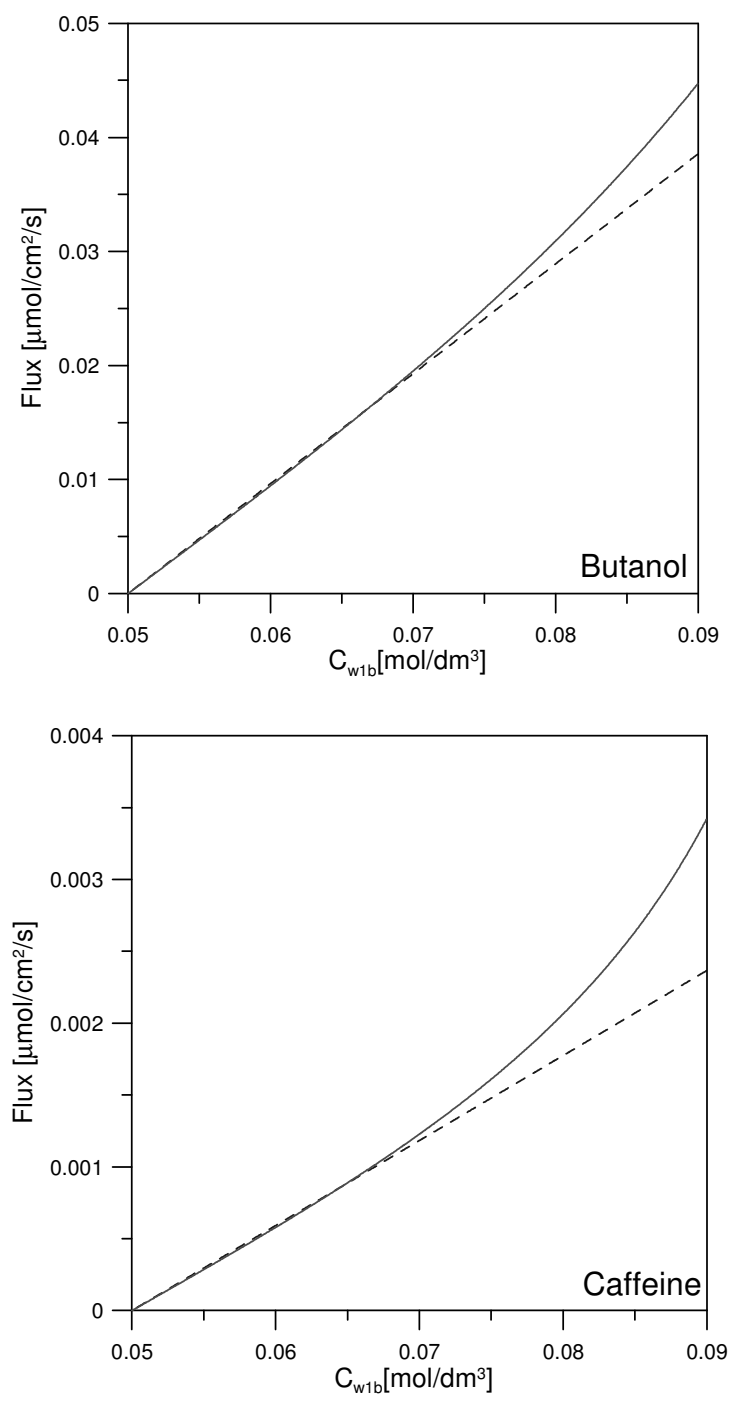

Fig. 3. The fluxes predicted by eq (10) (solid red line) and eq (11) (dashed blue line) for butanol and caffeine transport across octanol membrane. The parameters used in the calculations were collected in Table 1. It was assumed that the thickness of diffuse layer and membrane was ten times smaller than before $\left(h_{w}=20 \mu \mathrm{m}\right.$ and $\left.h_{o}=10 \mu \mathrm{m}\right)$. 


\section{REFERENCES}

[1] Q. Al-Awqati, Nature Cell Biology, 1999, $1201-202$.

[2] A. Missner and P. Pohl, Chem. Phys. Chem. 2009, 10, 1405.

[3] J.F. Nagle, J.C. Mathai, M.L. Zeidel and S. Tristram-Nagle, J. Gen. Physiol. 2008, 131 77-85.

[4] D.M. Miller, Biochimica et Biophysica Acta 1991, 1065, 75-81.

[5] R.H. Guy and D.H. Honda, International Journal of Pharmaceutics 1984, 19, 129-137.

[6] P.R. Fisk, M.G. Ford and P. Watson. in (Compton, R.G. and Hancock, G., eds.) Applications of kinetic modelling, Elsevier, Amsterdam 1999, pp. 161-194.

[7] G.L. Mosher, Pharmaceutical Research 1994, 11, 1325-1329.

[8] D.M. Miller, Biochimica et Biophysica Acta 1986, 856, 27-35.

[9] W.J. Albery, J.F. Burke, E.B. Leffler and J. Hadgraft, J. Chem. Soc. Faraday I 1976, 72, 1618-1626.

[10] S.T. Hwang, AIChE Journal 2004, 50, 862-870.

[11] C.A. Ward, R.D. Findlay and M. Rizk, Journal of Chemical Physics 1982, 76, 5599-5605.

[12] J.A.W. Elliot and C.A. Ward. in (Rudzinski, W., Steele, W.A. and Zgrablich, G., eds.) Equilibria and dynamics of gas adsorption on heterogeneous solid surfaces, Elsevier, Amsterdam 1997, pp. 285-334.

[13] C.A. Ward and G. Fang, Physical Review E 1999, 59, 429-440.

[14] W. Rudzinski and T. Panczyk, Journal of Physical Chemistry B 2000, 104, 9149-9162.

[15] F.K. Skinner, C.A. Ward and B.L. Bardakjian, Biophysical Journal 1993, 65, 618-629.

[16] P. Chen and A.W. Neumann, Colloids and Surfaces A 1998, 143, 331-338.

[17] W. Plazinski and W. Rudzinski, Langmuir 2009, 25, 298-304.

[18] W. Piasecki, Journal of Physical Chemistry B 2006, 110, 13138-13143.

[19] J. Hadgraft. in (Compton, R.G. and Hancock, G., eds.) Applications of kinetic modelling, Elsevier, Amsterdam 1999, pp. 121-132.

[20] K.D. McCarley and A.L. Bunge, Journal of Pharmaceutical Sciences 2001, 90,) 1699-1719.

[21] D.M. Miller, Biochimica et Biophysica Acta 1991, 1065, 69-74.

[22] S.B. Hladky, European Biophysical Journal 1987, 15, 251-255.

[23] P. Pohl, S.M. Saparov and Y.N. Antonenko, Biophysical Journal 1998, 75, 1403-1409. 